\title{
Interpreting deactivations in neuroimaging
}

\author{
Dave J. Hayes ${ }^{1 *}$ and Adrianne G. Huxtable ${ }^{2}$ \\ ' Mind, Brain Imaging and Neuroethics Research Unit, Institute of Mental Health Research, University of Ottawa, Ottawa, ON, Canada \\ 2 Department of Comparative Biosciences, University of Wisconsin, Madison, WI, USA \\ *Correspondence: david.hayes@theroyal.ca
}

Functional magnetic resonance imaging (fMRI) is a relatively young tool for understanding how the brain's activities contribute to physical and psychological processes. In the roughly 20 years since its introduction, fMRI has developed at an astounding rate regarding advances in both the acquisition of images, as well as improved post-acquisition analysis (Bandettini, 2011). Blood oxygenation level dependent (BOLD) activity is its key measure and most studies rely on contrasts between two conditions of interest (e.g., BOLD signal during exposure to painful stimuli $>$ signal during exposure to non-painful stimuli) to identify regions of functional significance. Alternately, electrophysiological techniques provide direct measures of neural activity in comparison to neuroimaging techniques. Deciphering the precise activity of the brain, in normal and pathological conditions, is of paramount importance; both neuroimaging and electrophysiological techniques will likely be crucial in this regard.

The BOLD signal has been thought of as a correlate of local synaptic activity (neuronal and possibly glial activity; e.g., Schummers et al., 2008), and recent studies using combined optogenetic imaging and fMRI have supported this central assumption (Lee et al., 2010). The hemodynamic response that underpins the BOLD signal is predominantly based on the displacement of deoxyhemoglobin by inflowing oxygenated hemoglobin. As such, BOLD signals rely heavily on blood volume and flow, as well as on oxygen consumption, all factors which are generally (but not always) positively correlated with neuronal activity (i.e., neurovascular coupling). (For more discussion on BOLD signal mechanisms, see Logothetis and Wandell, 2004.) While the underlying nature of positive BOLD responses is increasingly agreed upon, the mechanisms associated with negative BOLD responses (NBRs) are less clear. In particular, it is unclear whether the relationships between neuronal activity and
positive/NBRs are similar across various brain regions, especially between cortex and subcortex.

A recent article by Mishra et al. (2011) addressed the issue of whether changes in neuronal activity (measuring local synaptic changes with local field potentials, LFP, and broader neuronal firing rates with multi-unit activity, MUA) corresponded to changes in fMRI-related signals (i.e., BOLD, as well as independent measures of blood volume and flow). They questioned whether the relationship between neuronal activity and fMRI signals would be similar for the cerebral cortex and subcortical structures, such as the caudate-putamen and the thalamus. Looking at both spontaneous brain activity (using an animal model of human absence epilepsy) and stimulus-induced activity (using tactile whisker stimulation) in rats, they found that increased activations in the sensory cortex and thalamus were observed across both neuroimaging and electrophysiological methodologies. Alternately, however, deactivations in the caudate-putamen (noted with BOLD, $\mathrm{CBV}$, and $\mathrm{CBF}$ ) occurred in conjunction with increases (LFP) or reductions (MUA) in neuronal activation (though MUA reductions were still greater than baseline and so were not deactivations; as summarized in Figure 8 of their paper).

Given their findings, the authors suggest that neurovascular coupling (i.e., that neuronal activity correlates positively with changes in CBF) does not hold for some subcortical structures, such as the caudateputamen. While neuronal mechanisms are generally believed to drive most BOLD signals, they state that the dissociation in the caudate-putamen demonstrates that a primary decrease in neuronal activation is not the mechanism associated with the decreased BOLD signal in this instance. They suggest that a decrease in CBF (without a corresponding decrease in neural activity) is the most likely candidate mechanism, arising perhaps from high activity increases in nearby regions (the so-called "vascular steal" effect) or from local vasoconstriction. Also, the large increase in LFP coupled with the reduced mean neuronal firing rate (MUA) may be explained by strong striatal inhibitory neuronal activity, which would produce large changes in synaptic activity (LFP) but would generally inhibit cell firing (MUA).

This work advances our understanding of the neurovascular coupling of the BOLD signal in fMRI and warns against the overly simplistic assumption of functional homogeneity across brain regions, especially when comparing cortical and subcortical activations. If neurovascular coupling cannot be assumed in some subcortical regions, current interpretations of subcortical activations in fMRI studies may be called into question (e.g., caudate-putamen activations in reward studies may not be strictly related to net increases in neuronal activity). Nonetheless, the question of whether this issue is tied exclusively to subcortical (as opposed to cortical) regions, and if and how this affects NBR-related mechanisms, is unclear. Indeed, future studies should consider whether changes in subcortical BOLD activity (particularly NBR) reflect corresponding changes in neuronal activation and may require researchers to use a combination of techniques, such as that used in Mishra et al. (2011).

Other studies, however, suggest that cortical BOLD deactivations do not always correspond to electrophysiological measures. For instance, a recent study by Yuan et al. (2011) compared spatially coregistered electroencephalographic oscillations and BOLD signals in the human sensorimotor cortex during a finger tapping task. They found that while positive BOLD signals correlated well with the EEG data (they overlapped and were negatively correlated with alpha, 8-13 Hz, and beta, $13-30 \mathrm{~Hz}$, bands), NBRs did not - suggesting a differential coupling between neuronal activity and positive and negative BOLD signals. Moreover, at least one study has shown that some NBRs can be produced in non-neuronal tissue 
(e.g., large cerebral veins; Bianciardi et al., 2011). Alternately, other studies in rats have demonstrated that the negative changes in hemodynamic response (as seen using optical imaging, laser Doppler flowmetry, or fMRI BOLD) noted in cortical regions following tactile stimulation corresponded well with decreases in neuronal activation (using multi-unit recording; Boorman et al., 2010; Yin et al., 2011).

The data from Mishra et al. (2011) and others suggest to us that the observed differential neurovascular coupling is perhaps less an issue of cortical vs. subcortical function, and perhaps more related to an unclear understanding of the underlying mechanisms of fMRI BOLD signal deactivations. (Nonetheless, we must concede that some interesting differences may yet be uncovered between cortex and subcortex, given the comparatively smaller, more intricately defined, subcortical regions and their associated microvasculature.) This is underscored by the fact that most fMRI studies do not report their findings of BOLD deactivations, which may show greater variability across subjects compared to positive BOLD (e.g., Yin et al., 2011), and given the numerous factors which may contribute to the generation of NBR noted above.

Nonetheless, what is clear is that a large component of the NBR does appear to be related to neuronal activity, and positive and negative BOLD signals occur simultaneously and likely even overlap spatially (e.g., via different cortical layers; see Boorman et al., 2010). Given its importance in interpreting neuroimaging data, we would like to make three basic proposals aimed at helping further elucidate the role of BOLD deactivations: use consistent terminology and report all deactivations; consider using an independent baseline; further explore the link between neurotransmission and BOLD deactivations.

Reporting deactivations will be most useful if it is clear how they have been measured. "Negative BOLD response" is often used in reference to decreased signal changes compared to a resting state condition in which the subject is explicitly instructed to close their eyes or stare at a fixation cross without actively engaging in mental activity (see Northoff et al., 2010 for discussion). In contrast, the BOLD signals in most studies are derived from contrasts between two conditions (with one as a rela- tive control). In this case, "BOLD deactivation" refers to a relatively lower signal in one condition over another, and in the presence of an implicit global baseline specific to each experiment (thus, signal changes extracted from regions of interest cannot be quantitatively compared across studies). In this usage, NBRs are specific instances of BOLD deactivation, whereas not all deactivations are NBRs. Currently, these terms are sometimes used interchangeably (e.g., Yin et al., 2011).

While comparing signal changes across tasks is informative, including resting state periods (as an approximate measure of the brain's intrinsic activity) in the study design may be additionally useful (Northoff et al., 2010). In particular, including distinct resting state conditions (e.g., staring at a fixation cross or an eye's closed period) before, after, and possibly throughout the experiment(s) of interest may provide an independent baseline for comparison. One could then compare the signal changes in the condition of interest against both the control and resting state conditions to determine clearly whether the deactivations seen are NBRs, and may allow for further investigation of the potential context-dependence of neurovascular coupling regarding NBRs. This approach also allows the comparison of signal changes across experiments and the ability to look for potential baseline drift. Interestingly, while the approach of considering the baseline independently is standard throughout the electrophysiological literature (e.g., Yuan et al., 2011) it is less commonly used in fMRI experiments.

It is important to briefly underscore some of the conceptual issues when considering the use of a baseline in fMRI. For instance, some brain regions actually increase their activity during the so-called resting state (when alert subjects are instructed to relax and not focus on external or internal stimuli; e.g., while staring at a fixation cross or closing their eyes). A common example of such a resting state network is the default mode network (e.g., perigenual anterior cingulate and posterior cingulate cortices), which also shows deactivations when subjects actively perform tasks. Although fMRI resting state and animal electrophysiology studies on oscillatory networks (Zuo et al., 2010) strongly support the notion of intrinsic brain activity, an absolute definition may be challenging given that the brain is never truly at rest. The continual barrage of sensory and interoceptive information (which likely varies across experimental conditions) makes capturing a true resting state impossible. However, given its potential importance on global brain function, we recommend using a reasonable operational definition of this baseline activity in conjunction with the best available methods (e.g., fixation cross or eyes closed). Future studies should also consider using more quantitative $\mathrm{fMRI}$ measures such as those described recently by Pike (2011). Also, see Northoff et al. (2010) for further discussion of these conceptual and methodological issues.

Finally, as evidence suggests that BOLD signals are tightly coupled to synaptic activity, future studies should aim to characterize the relationships between BOLD activity and neurotransmission since this has the potential to dramatically shift views of brain activity in normal and pathophysiological functioning. In particular, studies in humans (using multimodal imaging, such as combining fMRI with magnetic resonance spectroscopy) and other animals (combining electrophysiological and/or imaging with neurochemical measures) should further explore the hypothesis that NBRs are related primarily to inhibitory post-synaptic potentials. Indeed, some recent studies in humans (Muthukumaraswamy et al., 2011) and rats (Boorman et al., 2010) suggest that the inhibitory neurotransmitter GABA may be a key mediator of the NBR.

In summary, Mishra and colleagues provided additional evidence of the complexity of fMRI BOLD activations and helped to raise a number of interesting questions, while also demonstrating the added value of combining technologies. To assist in answering these important questions, which affect nearly all fMRI studies, we suggest the consistent use of operationalized terms and the collection of independent baseline conditions. In addition, we recommend more studies which use translational and/or multimodal approaches, such as that used in Mishra et al. (2011), for better testing the hypothesis that NBRs are driven largely by decreases in neuronal activity related to inhibitory neurotransmission. The precise mechanisms of NBRs or deactivations must be fully understood for a complete and accurate interpretation of neuroimaging data. 


\section{ACKNOWLEDGMENTS}

Dave J. Hayes holds a Postdoctoral Fellowship from the Canadian Institutes of Health Research (CIHR). Adrianne G. Huxtable is supported by funding in the laboratory of G.S. Mitchell (NIH HL80209 and HL69064).

\section{REFERENCES}

Bandettini, P.A. (2011). Sewer pipe, wire, epoxy, and finger tapping: the start of fMRI at the Medical College of Wisconsin. Neuroimage doi: 10.1016/j.neuroimage.2011.10.044. [Epub ahead of print].

Bianciardi, M., Fukunaga, M., Van Gelderen, P., De Zwart, J. A., and Duyn, J. H. (2011). Negative BOLD-fMRI signals in large cerebral veins. J. Cereb. Blood Flow Metab. 31, 401-412.

Boorman, L., Kennerley, A. J., Johnston, D., Jones, M., Zheng, Y., Redgrave, P., and Berwick, J. (2010). Negative blood oxygen level dependence in the rat: a model for investigating the role of suppression in neurovascular coupling. J. Neurosci. 30, 4285-4294.

Lee, J. H., Durand, R., Gradinaru, V., Zhang, F., Goshen, I., Kim, D. S., Fenno, L. E., Ramakrishnan, C., and
Deisseroth, K. (2010). Global and local fMRI signals driven by neurons defined optogenetically by type and wiring. Nature 465, 788-792.

Logothetis, N. K., and Wandell, B. A. (2004). Interpreting the BOLD signal. Annu. Rev. Physiol. 66, 735-769.

Mishra, A. M., Ellens, D. J., Schridde, U., Motelow, J. E. Purcaro, M. J., Desalvo, M. N., Enev, M., Sanganahalli, B. G., Hyder, F., and Blumenfeld, H. (2011). Where fMRI and electrophysiology agree to disagree: corticothalamic and striatal activity patterns in the WAG/Rij rat. J. Neurosci. 31, 15053-15064.

Muthukumaraswamy, S. D., Evans, C. J., Edden, R.A., Wise, R. G., and Singh, K. D. (2011). Individual variability in the shape and amplitude of the BOLD-HRF correlates with endogenous GABAergic inhibition. Hum. Brain Mapp. 33, 455-465.

Northoff, G., Duncan, N. W., and Hayes, D. J. (2010). The brain and its resting state activity - experimental and methodological implications. Prog. Neurobiol. 92, 593-600.

Pike, G. B. (2011). Quantitative functional MRI: concepts, issues and future challenges. Neuroimage. doi: 10.1016/j.neuroimage.2011.10.046. [Epub ahead of print].

Schummers, J., Yu, H., and Sur, M. (2008). Tuned responses of astrocytes and their influence on hemodynamic signals in the visual cortex. Science 320, 1638-1643.
Yin, H., Liu, Y., Li, M., and Hu, D. (2011). Hemodynamic observation and spike recording explain the neuronal deactivation origin of negative response in rat. Brain Res. Bull. 84, 157-162.

Yuan, H., Perdoni, C., Yang, L., and He, B. (2011). Differential electrophysiological coupling for positive and negative BOLD responses during unilateral hand movements. J. Neurosci. 31, 9585-9593.

Zuo, X. N., Di Martino, A., Kelly, C., Shehzad, Z. E., Gee, D. G., Klein, D. F., Castellanos, F. X., Biswal, B. B., and Milham, M. P. (2010). The oscillating brain: complex and reliable. Neuroimage 49, 1432-1445.

Received: 09 January 2012; accepted: 23 January 2012; published online: 07 February 2012.

Citation: Hayes DJ and Huxtable AG (2012) Interpreting deactivations in neuroimaging. Front. Psychology 3:27. doi: 10.3389/fpsyg.2012.00027

This article was submitted to Frontiers in Cognitive Science, a specialty of Frontiers in Psychology.

Copyright (c) 2012 Hayes and Huxtable. This is an openaccess article distributed under the terms of the Creative Commons Attribution Non Commercial License, which permits non-commercial use, distribution, and reproduction in other forums, provided the original authors and source are credited. 